Mémoires du livre

Studies in Book Culture

\title{
« J'en suis venu à accepter aussi bien l'autorité des récits et celle des articles »
}

\section{Le pulp Adventure et la circulation des imaginaires sériels dans les imaginaires sociaux}

\section{Matthieu Letourneux}

Volume 10, numéro 1, automne 2018

Voyager dans la modernité : des moyens techniques aux supports imprimés de l'aventure

Adventure: From Travel Technology to Print Media

URI : https://id.erudit.org/iderudit/1055406ar

DOI : https://doi.org/10.7202/1055406ar

Aller au sommaire du numéro

\section{Éditeur(s)}

Groupe de recherches et d'études sur le livre au Québec

ISSN

1920-602X (numérique)

Découvrir la revue

Citer cet article

Letourneux, M. (2018). « J'en suis venu à accepter aussi bien l'autorité des récits et celle des articles " : le pulp Adventure et la circulation des imaginaires sériels dans les imaginaires sociaux. Mémoires du livre / Studies in Book Culture, 10(1). https://doi.org/10.7202/1055406ar

\section{Résumé de l'article}

Dans les pulps américains, les imaginaires sériels débordent l'espace de la fiction pour contaminer la partie rédactionnelle du magazine. Le pulp Adventure, spécialisé dans les récits d'aventures, illustre cette circulation des imaginaires. À travers des rubriques comme « The Camp-Fire » et « Ask Adventure ", il invite les consommateurs à échanger avec auteurs et spécialistes, créant une communauté de lecteurs liés entre autres par des projets tout droit sortis de récits d'aventures et manifestant une forme de fictionnalisation de l'existence. Or, dans ces fantasmes d'accomplissement romanesque peut se lire une reformulation du rêve pionnier, au moment même où celui-ci s'efface aux États-Unis pour laisser place à de nouveaux modèles de réussite sociale. À partir de cet exemple, nous voudrions montrer plus largement que, dans leur façon de structurer les imaginaires et de les associer à des formes identifiables, les genres permettent d'interroger la manière dont une culture donnée organise en systèmes de signification et à travers des modèles récurrents de narration certaines des préoccupations d'une collectivité.

Tous droits réservés ( Groupe de recherches et d'études sur le livre au Québec, 2019 


\title{
MÉMOIRES DU LIVRE \\ STUDIES IN BOOK CULTURE
}

\section{« J'EN SUIS VENU À ACCEPTER AUSSI BIEN L'AUTORITÉ DES RÉCITS ET CELLE DES ARTICLES » :} Le pulp Adventure et la circulation des imaginaires sériels dans les imaginaires sociaux

\author{
Matthieu LETOURNEUX \\ Université Paris-Nanterre
}

Dans les pulps américains, les imaginaires sériels débordent l'espace de la fiction pour contaminer la partie rédactionnelle du magazine. Le pulp Adventure, spécialisé dans les récits d'aventures, illustre cette circulation des imaginaires. À travers des rubriques comme "The Camp-Fire» et "Ask Adventure», il invite les consommateurs à échanger avec auteurs et spécialistes, créant une communauté de lecteurs liés entre autres par des projets tout droit sortis de récits d'aventures et manifestant une forme de fictionnalisation de l'existence. Or, dans ces fantasmes d'accomplissement romanesque peut se lire une reformulation du rêve pionnier, au moment même où celui-ci s'efface aux États-Unis pour laisser place à de nouveaux modèles de réussite sociale. À partir de cet exemple, nous voudrions montrer plus largement que, dans leur façon de structurer les imaginaires et de les associer à des formes identifiables, les genres permettent d'interroger la manière dont une culture donnée organise en systèmes de signification et à travers des modèles récurrents de narration certaines des préoccupations d'une collectivité.

In American pulps, serial imaginaries overflowed the fictional space to contaminate the editorial component of the magazine. The pulp Adventure, which specialized in adventure stories, illustrates this circulation of the imaginary among fictional and non-fictional texts. Through sections such as "The Camp-Fire" and "Ask Adventure," it invited consumers to interact with authors and specialists, creating a 
community of readers bound by projects which seemed to come straight out of adventure stories, creating a form of fictionalisation of existence. In these fantasies of romantic achievement, we can read a reformulation of the pioneering dream at the very moment when it was being eroded in the United States and being replaced by new models of social success. Building from this case study, we would like to show more generally that, in their way of structuring imaginations and associating them with identifiable forms, popular genres make it possible to question the way in which a given culture organizes certain community concerns into systems of meaning through identifiable narrative models.

Au début $\mathrm{du} \mathrm{xx}^{\mathrm{e}}$ siècle, le système éditorial américain délaisse le format des dime novels pour celui des $p u l p s^{1}$, magazines de fiction très bon marché le plus souvent spécialisés dans un genre (aventure, western, policier, sentimental...) et offrant dans chaque numéro un roman à suivre, un ou plusieurs petits romans (novelettes) et une série de nouvelles, l'ensemble étant généralement complété par des articles et rubriques sur des sujets trouvant un écho avec les thèmes privilégiés dans le magazine. L'un des pulps les plus fameux et les plus anciens est $A_{d v e n t u r e}{ }^{2}$, spécialisé (comme son nom l'indique) dans l'aventure. Comme beaucoup de pulps, il propose des rubriques non fictionnelles qui laissent une place importante à l'échange avec les lecteurs. Ce sont ces rubriques que nous voudrions étudier ici pour comprendre quelle signification a pu prendre l'imaginaire de l'aventure à l'époque, et comment il a pu déborder les limites du roman pour nourrir l'imagination des lecteurs. En effet, à travers ces pages, se révèle une circulation très particulière des thèmes entre espaces fictionnels et non fictionnels, qui nous informe sur la façon dont les productions sérielles peuvent irriguer les imaginaires sociaux.

\section{«The Camp-Fire » et « Ask Adventure » ou l'aventure hors de la fiction}

Lancé en 1910 par la Ridgway Publishing Company, Adventure est l'un des pulps les plus importants de l'entre-deux-guerres. Fait rare dans ce marché éditorial où les titres font souvent faillite en quelques années, le magazine a bénéficié d'une longévité exceptionnelle, puisqu'il ne cesse de paraître qu'en 1971. Il reste longtemps l'un des pulps les plus réputés, avec The Argosy, Blue Book ou All-Story. Son succès lui vaut d'ailleurs d'être présenté en 1935 par le Time Magazine comme étant « le pulp le plus important ${ }^{3}$ ». Le périodique publie des auteurs jouissant d'un certain prestige, comme Talbot Mundy, W. C.

Vol. 10, n 1 | Automne 2018

«Voyager dans la modernité : des moyens techniques aux support imprimés de l'aventure » 
Tuttle, Hugh Pendexter, Harold Lamb, Rafael Sabatini, Henry Bedford-Jones, etc., tous spécialisés dans les récits d'aventures au sens le plus large du terme, du western aux aventures exotiques en passant par les aventures historiques. À côté de ces récits constituant l'essentiel du magazine, quelques rubriques offrent un rendez-vous régulier aux lecteurs.

C'est en 1912 que les éditeurs lancent la première de ces rubriques, "The Camp-Fire », qui se pense comme un lieu d'échange entre les lecteurs et les différents acteurs du magazine, rédacteurs et romanciers. Les lecteurs peuvent non seulement y poser des questions aux auteurs, mais également aux autres lecteurs, qui sont invités dans ce cas à répondre dans les numéros suivants. De leur côté, les écrivains peuvent commenter les récits qu'ils ont publiés dans le numéro, souvent pour apporter des informations complémentaires sur les œuvres. Enfin, les nouveaux auteurs peuvent se présenter, via une biographie plus ou moins longue - le plus souvent de quelques paragraphes, parfois d'une ou deux pages. L'espace de «The Camp-Fire » est également ouvert aux commentaires. Ainsi le journal reproduit-il régulièrement des critiques sur les œuvres formulées par les lecteurs, en particulier lorsqu'il s'agit de discuter la véracité de certaines affirmations. Dans le numéro d'août 1923, par exemple, un lecteur se gausse de la manière dont l'auteur Albert Richard Wetjen décrit l'usage du tabac à priser chez les Suédois, et dont il suppose en outre que ceux-ci sont toujours prêts à boire un verre quand on le leur propose $e^{4}$. Ailleurs, un lecteur scrupuleux remarque que les feux vert et rouge sont trop loin l'un de l'autre sur les bateaux pour se refléter tous deux sur le visage d'un des personnages, comme l'indique un récit ${ }^{5}$. Un troisième lecteur regrette la censure qu'opère le périodique sur les jurons des personnages, soulignant qu'ils participent de l'effet de réel ${ }^{6}$. À l'inverse, quand un auteur se fait attaquer dans un essai pour les qualités factuelles d'un de ses récits (comme ce fut le cas pour « North of 36 », un récit d'Emerson Hough), c'est encore dans «The Camp-Fire » qu'on convoque une série d'experts pour prendre sa défense ${ }^{7}$. Ainsi, les lecteurs sont invités à participer à la vie du périodique et aux débats qu'il suscite, suivant une logique visant à produire une communauté d'intérêt qui dépasse la seule consommation des fictions.

Face au succès du « Camp-Fire », et sans doute sensibles au caractère souvent spécialisé des questions posées par les lecteurs, les éditeurs décident en 1917 de le compléter d'une seconde rubrique, «Ask Adventure». Le fonctionnement de celle-ci est très particulier, puisque l'éditeur propose à ses 
lecteurs de s'adresser directement à une série de spécialistes, identifiés par leur nom et leur domaine de compétence dans une liste de plus en plus longue au fil du temps (ils seront une centaine au plus fort du succès de la rubrique) ${ }^{8}$ : spécialistes des différentes régions du globe, des bateaux à moteur, des armes à feu, des animaux, du système ferroviaire, de la chasse, de la pêche, etc. Chaque semaine, une sélection de questions et de réponses est publiée. Cellesci peuvent toucher à des sujets très différents : il peut s'agir de demander des précisions sur une figure historique ou un événement marquant (un épisode de l'épopée de l'Ouest, une expédition fameuse), de solliciter des conseils pour une excursion en canoë sur le Mississippi ${ }^{9}$, de s'enquérir de la meilleure façon de conserver les peaux de serpent ${ }^{10}$, de savoir ce qu'il faut emporter pour aller chasser le wallaby en Nouvelle-Guinée ${ }^{11}$, ou de connaittre les conditions à remplir pour aller chercher de l'or dans un pays lointain ${ }^{12}$. À toutes ces questions, les experts répondent, avec le plus grand sérieux, par de longs développements, des conseils détaillés pour le voyage et des suggestions de lecture.

$\mathrm{Si}$ «Ask Adventure » et «The Camp-Fire » sont restés le noyau de la partie rédactionnelle ${ }^{13}$, d'autres rubriques ont pu apparaitre pour un temps bref. C'est le cas par exemple de «Lost Trails », dans laquelle les lecteurs pouvaient faire appel aux autres lecteurs pour retrouver des connaissances perdues de vue. Un autre service offert était celui des «identification cards》, sortes de cartes d'identité, en papier ou métal (pour un supplément de 25 cents), permettant, par un système d'envoi dans lequel la rédaction d'Adventure jouait le rôle d'intermédiaire, de prévenir les proches du possesseur de la carte en cas de mort ou d'accident grave lors d'un voyage. Évoquons également un dispositif favorisant la correspondance des soldats américains et canadiens pendant la Première Guerre mondiale, une bourse d'échange pour les numéros manquants du magazine, une autre invitant les lecteurs à transmettre à la rédaction les chansons de marins et de pionniers pour qu'elles soient imprimées dans les pages du magazine, et surtout une organisation complexe de «Camp-Fire Stations » permettant aux lecteurs du pulp d'être logés partout dans le monde par d'autres lecteurs, jusqu'à former une "Adventure Travel Association ${ }^{14} \gg-$ le principe étant redoublé par tout un système de badges et d'insignes présentés comme des signes de reconnaissance ${ }^{15}$.

On le voit, tous ces dispositifs étaient destinés à concrétiser la « communauté de lecteurs » et ainsi à réduire la distance qu'introduit nécessairement toute 
production médiatique entre les émetteurs et les récepteurs, a fortiori dans des journaux qui, comme les pulps, offrent essentiellement des fictions, c'est-àdire des textes entretenant une relation au monde plus faible que les articles d'actualité ${ }^{16}$. C'est cette fonction que remplissaient toutes ces rubriques qui insistaient sur la volonté des éditeurs de se rendre utiles aux lecteurs et de jouer un rôle d'intermédiaires entre différents interlocuteurs (auteurs et lecteurs, lecteurs entre eux, lecteurs et experts). Autrement dit, il s'agissait de prétendre dépasser la dimension commerciale du périodique et la distance propre à la communication littéraire, pour leur substituer une forme de communauté de pairs. Certes, cette communauté était largement construite, et n'excédait pas, pour la plupart des acheteurs, le temps de la lecture du périodique, mais elle n'en était pas moins extrêmement productive en termes communicationnels. En effet, la camaraderie associée à l'aventure, la logique d'entraide mise en avant, le prétendu désintéressement des éditeurs ${ }^{17}$, la dimension initiatique que portait en lui le système d'experts et de nouveaux venus s'articulaient pour reformuler dans la communication éditoriale un ensemble de valeurs caractéristiques de l'aventure romanesque (ce qu'indiquait d'ailleurs le titre de la rubrique « Camp-Fire», entre retrouvailles de pionniers et jamboree scout). À travers ce dispositif, le périodique pouvait ainsi inviter les consommateurs à redéfinir leur relation au magazine suivant une logique d'engagement qui convertissait la communauté de lecteurs en camaraderie aventureuse. C'est ce que montre bien le texte de présentation du «Camp-Fire», jouant sur cette fraternité des auteurs et des lecteurs: « Nous sommes liés entre nous par un même amour pour les choses fortes et saines qui touchent au plein air, pour les discours venus des confins du monde, pour les hommes définis par leurs actes et non prisonniers des événements. L'esprit d'aventure vit dans chacun des hommes, le reste est affaire de fortune ${ }^{18}$. »

On voit combien la logique du magazine et la mixité des textes qu'elle permet produit un écart considérable par rapport au système des collections populaires qui se développe au même moment en France. En effet, si les productions de Tallandier, Ferenczi ou Fayard sont largement diffusées dans les réseaux de distribution de la presse, elles le sont suivant le principe de la série de volumes qui les conduit à abandonner le terrain du discours d'actualité (dont on ne trouve plus trace que de façon oblique dans la fiction). Ce n'est pas le cas des pulps, qui conservent une dimension journalistique, non seulement à travers la présence de rubriques non fictionnelles, mais aussi via 
d'autres caractéristiques de la presse : polyphonie des rédacteurs sur un même support, postures discursives et non pas seulement narratives des auteurs des textes dans "The Camp-Fire", exhibition de la communication réelle (généralement dissimulée dans les fictions au profit d'un modèle narrateur/narrataire), projets concrets et informations factuelles, toutes ces caractéristiques ont une incidence sur les fictions elles-mêmes et sur la manière dont le lecteur est invité à les appréhender. En effet, la communication tend à inscrire les récits dans une dynamique médiatique insistant sur le contexte de production et engageant l'actualité ou la relation au monde. C'est d'autant plus vrai que les échanges entre rédacteurs et lecteurs sont loin de porter uniquement sur les fictions, puisqu'ils s'ouvrent à des questions sur les pays lointains, sur l'Histoire, les armes à feu, les conditions de voyages, les difficultés pratiques auxquelles sont notamment confrontés des chercheurs d'or, des négociants ou des éleveurs de bétail. Ils mettent du même coup en jeu des problèmes économiques, géopolitiques ou culturels. Autrement dit, ils ressaisissent le monde de l'aventure, habituellement pensé en termes de déliaison radicale, dans la complexité des échanges collectifs qui caractérisent notre expérience quotidienne.

Les parties rédactionnelles tendent à ancrer les fictions dans le réel et à inviter le lecteur à tisser des liens avec l'actualité, d'une façon similaire à celle qui prévaut dans le feuilleton publié dans la presse au xix ${ }^{\mathrm{e}}$ siècle $^{19}$. Mais contrairement à ce qui se produit dans la culture médiatique du xix ${ }^{\mathrm{e}}$ siècle, l'ensemble des discours publiés dans les pulps, fictionnels ou non, sont liés entre eux par une unité générique qui les constitue en série cohérente. Dans le cas d'Adventure, c'est l'imaginaire de l'aventure qui assure le lien entre fictions et parties rédactionnelles, avec pour thèmes communs le voyage, les pays lointains, l'engagement dans l'inconnu, le refus du quotidien et, plus largement, les traits caractéristiques de cette mystique de l'aventure décrite par Sylvain Venayre ${ }^{20}$. Mais, ailleurs, ce seront d'autres unités génériques, comme dans les pulps de science-fiction (qui offrent des articles de vulgarisation scientifique), dans les westerns (avec bourses d'échanges d'objets indiens, descriptions de lieux mythiques de l'Ouest ou articles sur les armes à feu), ou dans les pulps sentimentaux (dans lesquels les fictions sont complétées par des petites annonces matrimoniales ou des rubriques d'horoscope). 


\section{Rêves de pionniers et principe de réalité}

Ainsi les pulps développent-ils une conception du genre qui dépasse le seul espace de la fiction pour caractériser un ensemble plus large de textes englobant également des productions non fictionnelles. En procédant de la sorte, ils supposent que l'amateur de westerns, de récits sentimentaux ou, ici, de romans d'aventures, n'aime pas uniquement un type de récits, mais aussi un certain nombre de sujets représentant en quelque sorte le noyau réel de son attrait pour le genre. Cette manière de procéder nous incite à penser que le genre ne correspond pas seulement à un ensemble de stéréotypes thématiques et narratifs, mais qu'il trouve de surcroitt un écho direct dans une vaste gamme de préoccupations et d'intérêts définissant une conception de l'existence; autrement dit, il trouve un écho dans notre manière de considérer le monde. Ici, le goût pour le roman d'aventures est lié plus largement à une "culture de l'aventure ", mais au même titre qu'il existerait une culture de la science-fiction, du sentimental, du western. C'est l'insistance, dans l'économie du magazine, sur une unité des imaginaires par-delà les frontières de la fiction qui produit ce qu'on pourrait appeler une « culture sérialisée globale », laquelle prend en particulier la forme d'une série de discours contaminés par la fiction. Dès leur frontispice, les rubriques «Ask Adventure» et "Camp-Fire» cherchent d'ailleurs à produire cet effet. Les illustrations d'« Ask Adventure » présentent en effet généralement un cowboy écrivant au journal; quant à celles de «The Camp-Fire», elles mettent en scène, suivant les numéros, explorateurs, guerriers des steppes ou trappeurs.

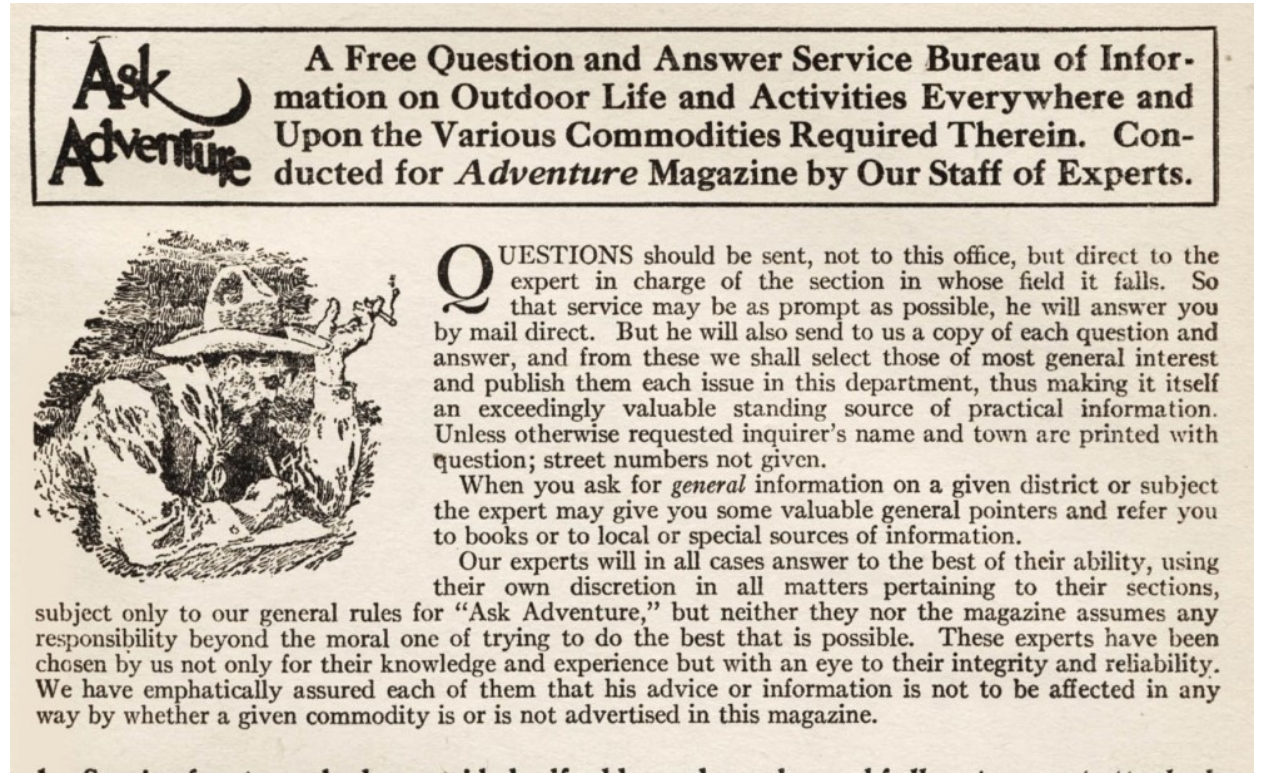

Vol. 10, n 1 | Automne 2018

«Voyager dans la modernité : des moyens techniques aux support imprimés de l'aventure » 


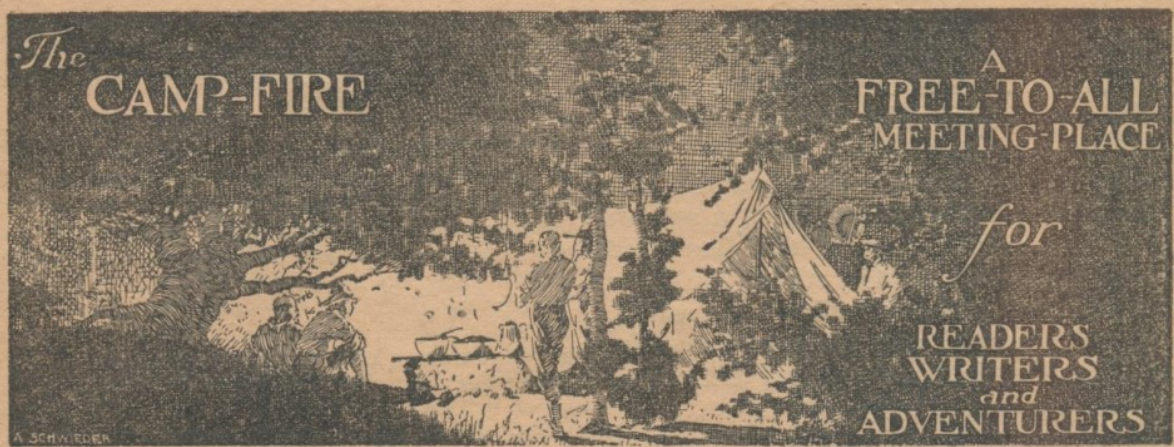

Our Camp-Fire came into being May 5, 1912, with our June issue, and since then its fire has never died down. Many have gathered about it and they are of all classes and degrees, high and low, rich and poor, adventurers and stay-at-homes, and from all parts of the earth. Some whose voices we used to know have taken the Long Trail and are heard no more, but they are still memories among us, and new voices are heard, and welcomed.

We are drawn together by a common liking for the strong, clean things of out-ofdoors, for word from the earth's far places, for man in action instead of caged by circumstance. The spirit of adventure lives in all men; the rest is chance.

But something besides a common interest holds us together. Somehow a real comradeship has grown up among us. Men can not thus meet and talk together without growing into friendlier relations; many a time does one of us come to the rest for facts and guidance; many a close personal friendship has our Camp-Fire built up between two men who had never met; often has it proved an open sesame between strangers in a far land.

Images 1 et 2 : Frontispices des rubriques « Ask Adventure » et « The CampFire » d'Adventure.

L'illustrateur, les sujets privilégiés et le vocabulaire graphique sont les mêmes que ceux des vignettes offertes en ouverture de chaque récit, manifestant l'unité recherchée entre les récits imaginaires et la manière d'envisager le monde.

Dans la dynamique du journal s'opère plutôt un glissement de la fiction au monde, dans la mesure où, pour le lecteur, atteindre les dernières pages du magazine après avoir lu une série de récits extraordinaires, équivaut à dériver des rêveries sur l'aventure romanesque vers l'aventure réelle. Et cette aventure réelle l'engage d'autant plus qu'elle concerne ses pairs, c'est-à-dire les autres lecteurs et les rédacteurs du journal. C'est donner l'impression que l'aventure n'est pas tout à fait hors de portée, et que les fictions ont une vocation exemplaire, invitant, ne serait-ce que fantasmatiquement, à une forme de participation. Autrement dit, ce que manifeste la structure du journal dans sa totalité, c'est cette possible vocation des fictions sérielles à organiser une certaine manière d'envisager l'existence dans le monde par-delà la fiction, mais par la médiation de celle-ci. 
C'est en tout cas ce que semblent indiquer les projets des lecteurs d'« Ask Adventure », puisqu'ils entrent directement en dialogue avec les récits. Ainsi en est-il des diverses expéditions pour aller chercher de l'or, si souvent préparées dans les pages d'« Ask Adventure »21, qui se placent dans les pas de Jack London, James Oliver Curwood et Stewart Edward White, trois figures prototypiques du roman d'aventures américain 22 - et, de fait, la ruée vers l'or fournit le sujet de bien des textes publiés dans $A d v e n t u r e^{23}$. De même, les récits narrant les exploits de héros voyageant de par le monde trouvent leur écho dans les lettres évoquant des idées de safari en Afrique ou d'emplois improbables dans les mers du Sud ${ }^{24}$. On retrouve dans ce type de projets la manière dont le roman d'aventures à l'américaine reconfigure dans une version individualiste les imaginaires coloniaux des récits européens ${ }^{25}$. C'est d'autant plus vrai que, loin de conter les exploits de colons dominateurs ou de sportsmen dilettantes, comme le faisaient les romanciers du xix ${ }^{\mathrm{e}}$ siècle, les auteurs d'Adventure offrent des récits centrés sur les mésaventures de personnages peu fortunés, qui gagent leur avenir dans des entreprises périlleuses dans l'espoir de gagner leur vie : tenter sa chance sur un navire en bout de course ${ }^{26}$, pêcher des perles sur une embarcation de fortune ${ }^{27}$, lutter contre une mutinerie sur un navire marchand ${ }^{28} \ldots$, ce sont de telles entreprises dans lesquelles se joue le destin d'un personnage qu'imitent les lecteurs à travers leurs projets.

Mais c'est surtout autour de l'imaginaire de l'Ouest que s'opère le plus nettement la circulation entre fiction et réalité. Ce n'est pas étonnant quand on songe que l'identité américaine s'est largement structurée autour de la figure des pionniers, de la frontier et de l'esprit d'aventure qui leur est associé (le fameux «Go West young man » qui a pu valoir au xix siècle pour projet politique ${ }^{29}$. Or, un bon tiers des récits du périodique portent sur le far west, lequel, dans la partie rédactionnelle, fournit une portion importante des sujets traités. Par rapport aux autres thématiques, l'imaginaire de l'Ouest possède un statut particulier, puisque, dans les années 1910-1920, il se situe à mi-chemin entre l'Histoire et les imaginaires contemporains. Bien des Américains ont le sentiment d'avoir connu ce monde, même s'il a déjà basculé dans un passé mythique. C'est pour cette raison qu'il est le plus souvent évoqué selon les modalités de la mémoire (quand bien même elle serait largement construite). Dans les articles du «Camp-Fire», cela se traduit par une insistance des romanciers sur les souvenirs personnels ou familiaux renvoyant à cet Ouest révolu ${ }^{30}$. En retour, des lecteurs font part de leur propre expérience, parfois 
pour corriger des erreurs d'auteurs. C'est le cas quand un lecteur prétend se fonder sur son expérience de la frontier ("I have known the border from New Mexico to Brownsville, Texas, for the last 30 years») pour affirmer qu'il n'a jamais vu d'homme de loi porter deux «long-barreled colts» à la fois, comme on les représente fréquemment dans les récits de l'Ouest ${ }^{31}$. Un autre, «somewhat familiar with the South-West frontier », qui dit avoir travaillé pour le sheriff de Grant County, certifie n'avoir jamais entendu parler d'un homme en frappant un autre d'un coup de crosse ${ }^{32}$. Ces exemples montrent bien que la relation à l'Histoire est entièrement médiatisée par la fiction (l'Ouest est affaire de sheriffs, de gunfights, de colts et de coups de crosse) et se définit par ses intertextes romanesques.

Mais l'Ouest structure également les imaginaires contemporains. De fait, il nourrit en profondeur les projets aventureux d'«Ask Adventure». Les lecteurs déplacent le rêve du cowboy dans un pays lointain en imaginant se lancer dans l'élevage en Tchécoslovaquie ou au Brésili3, ou encore quand ils parlent de camper dans les Rocheuses, de descendre une rivière en canoë34, de voyager dans le Grand Nord canadien en vivant de chasse ${ }^{35}$, ou d'explorer à moto le Mexique pittoresque ${ }^{36}$. Tous ces exploits imaginés établissent une sorte de continuité entre l'épopée du far west et les ambitions des nouveaux aventuriers.

En réalité, l'esprit de la frontier souffle sur la plupart des échanges qui nourrissent la correspondance d'«Ask Adventure », même quand il s'agit d'exploiter une mine dans le Mojave ou d'aller faire fortune en Afrique. S'installer dans une région sauvage, ne pas craindre l'adversité, faire preuve de courage et conquérir une place dans le monde grâce à sa détermination, c'est là un vocabulaire récurrent des lecteurs et des rédacteurs dont la source se trouve dans les actions des héros des récits de l'Ouest. C'est également par l'imaginaire de la conquête américaine que se comprend la façon dont le roman d'aventures américain redéfinit les modèles français et anglais du genre, à travers la mise en scène d'un héros individualiste calqué sur le modèle du cowboy et du pioneer (plutôt que sur celui de ces militaires, ingénieurs ou serviteurs de l'Empire des romans européens), et gagnant par la force sa place dans le monde, en marge de la loi.

Même quand les questions d'« Ask Adventure » portent sur l'organisation de voyages de loisirs, seul, entre amis ou en couple, se perçoit la trace de cet 
imaginaire individualiste. Il s'agit toujours de sortir des sentiers battus, et de partir le plus loin possible des hommes. Souvent, l'expert d'Adventure doit se lancer dans un travail de démystification des projets romanesques, par exemple quand il explique à un lecteur qu'il n'est pas besoin d'emporter d'arme à feu pour un séjour dans les îles canadiennes puisque les principales bêtes sauvages sur place sont les moustiques ${ }^{37}$. À l'inverse, il faudra raisonner un automobiliste en herbe décidant de voyager de Nome (Alaska) au Cap Horn, en lui signalant le peu de sérieux du projet étant donnée l'absence de routes - à moins de disposer d'une autochenille et... de quelques décennies pour le mener à terme ${ }^{38}$. On est de même un peu perplexe (comme l'est d'ailleurs l'expert) quand tel lecteur, qui reconnait ne jamais avoir quitté les latitudes de New York et de Boston, demande s'il est possible de partir seul et sans guide vivre deux ans en autosubsistance dans le Sud-Nigéria, sans connaître la faune et la flore, le coût de la vie, celui du voyage, et s'en remettant pour finir totalement aux experts d'«Ask Adventure» («any other useful information you can give me would be greatly appreciated 39 »). Ce type d'échanges, très fréquent, entre des lecteurs à l'imagination romanesque et des experts plus réalistes douchant leur enthousiasme ${ }^{40}$, montre combien l'imaginaire des œuvres pouvait contaminer la relation au monde d'une manière parfois fort éloignée de la réalité. Car ces contraintes concrètes que rappellent les experts sont celles (financières, matérielles, juridiques, etc.) qu'occultent le plus souvent les romans d'aventures au profit d'événements plus sensationnels.

Dépouillés du flou de l'imagination romanesque, les messages d'« Ask Adventure » se dévoilent comme autant de projets dont l'enthousiasme naif n'a d'égal que l'étonnante méconnaissance du terrain. C'est dire la dimension donquichottesque que porte en lui le pacte de lecture du périodique, comme si les lecteurs peinaient à prendre la mesure de l'écart qui sépare la fiction de l'existence. Et, de fait, les lecteurs se mettent en scène en singeant les postures et les discours de personnages de romans, adoptant un parler hardboiled et une attitude virile typiques des fictions d'aventures américaines ${ }^{41}$, parfois jusqu'au pastiche des intrigues de genre, comme lorsqu'un voyage est décrit comme une conquête de l'Ouest : «A chum and I are going to make a trip through the West this summer. I have had some experience touring and camping. I built up an old Ford to make several trips in the East. The territory we wish to invade is new to me ${ }^{42}$. " On voit combien, par-delà les projets, les postures, l'expression et, plus largement, les imaginaires sont contaminées par la fiction. 
Mais, si les lecteurs peuvent apparaitre comme les dupes des récits qui leur sont offerts, la partie rédactionnelle du magazine n'est pas étrangère à cet effet. Lorsqu'il s'agit d'évoquer l'œuvre, celle-ci est systématiquement abordée en évacuant les questions d'écriture, et en ressaisissant non seulement la fiction, mais toute la communication littéraire, à travers l'illusion référentielle d'une aventure réelle qui en serait la vérité. Ainsi, les nouveaux auteurs qui, comme le veut la tradition, se présentent aux lecteurs dans les pages du «Camp-Fire » n'évoquent presque jamais les autres récits qu'ils ont publiés, leur conception de la littérature ou leur vocation d'écrivain, mais presque toujours leurs aventures passées ou leurs faits d'armes durant la Première Guerre mondiale ${ }^{43}$, ici encore avec un langage hardboiled qui tend à les confondre avec leurs personnages ${ }^{44}$. Mais, en réalité, c'est toute la scénographie auctoriale associée au périodique qui parait empruntée aux fictions d'aventures ${ }^{45}$. Ainsi, un auteur comme Barry Scobee se présente-t-il à travers une anecdote qui semble tout droit sortie d'un roman : «Here's my luck. I was an attendant in a Keely Cure institute once. No I wasn't working my way through. Staying in a room one night to watch a dopey, I was awakened from profound sleep by bim standing over me brandishing a razor and a revolver and calling me the man who had run away with his wife $e^{46}$. » Et ce sont des souvenirs tout aussi romanesques que met en avant Walter Farnham : « it is suggested that I give you my adventures. Well - I have been shot at twice, and missed, by different men, being unarmed both times myself, and once a wild Italian tried to split me with his knife ${ }^{47}$. » Le modèle imaginaire qui sert de fondement à la scénographie auctoriale est tout simplement celui du héros de roman d'aventures, affaiblissant la barrière entre l'auteur et l'œuvre.

De la même façon, les auteurs qui parlent de leur œuvre ne le font presque jamais en termes d'écriture. Non seulement ceux-ci ne semblent jamais se préoccuper de style, mais même la question des effets est absente des propos du « Camp-Fire ». Ce qui importe avant tout, c'est d'insister sur les sources authentiques de l'anecdote ${ }^{48}$. Le ton hardboiled, la mise en scène du passé aventureux de l'auteur et l'insistance sur le caractère véridique des anecdotes à l'origine des récits redéfinissent trois des grands pôles structurants de la fiction - le style, l'auteur et l'intrigue - à l'aune d'une réalité référentielle omniprésente, et ces gages de véridicité inlassablement réaffirmés sont autant d'invitations à saisir les œuvres dans leur relation au monde.

Les conséquences de cette logique de contamination réciproque de la fiction et du monde sont exprimées de manière synthétique par un lecteur du 
magazine :

Avec le recul, et à la réflexion, j'en viens à me dire qu'Adventure a contribué dans une large mesure à mon éducation. N'ayant pas pu voyager autant que je l'aurais voulu, je me rends compte que je suis devenu familier des us et coutumes des peuples exotiques. Je suis un lecteur attentif du «Camp-Fire» et des autres rubriques d'Adventure. Et comme les discussions et débats sont menés par des hommes qui peuvent se fonder sur leur expérience propre, j'en suis venu à accepter aussi bien l'autorité des récits que celle des articles ${ }^{49}$.

Les conventions de la fiction peuvent bien reposer sur la dynamique sérielle du genre, elles peuvent bien obéir à des règles commandées par des architextes fictionnels ${ }^{50}$, elles n'en engagent pas moins une manière de dire le monde et, à travers elle, une manière pour le lecteur de le considérer.

\section{L’aventure comme espace de négociation imaginaire}

Quel est ce discours qui se fait jour à l'articulation de la fiction et des articles? Pour le comprendre, on peut examiner un troisième type de documents, certes moins spécifique à Adventure, mais qui entre ici encore en résonance avec les autres types de textes : les publicités. On notera d'abord que la nature des publicités semble indiquer que le public d'Adventure était populaire. Certes, à cette époque où s'imposent progressivement aux États-Unis des modes de consommation modernes, se rencontrent déjà couramment des publicités pour des cigarettes, du chewing gum ou des montres bon marché. Mais la plus grande part des annonces concerne des écoles de formation professionnelle : «Home Study Business Courses», «International Correspondance Courses», «Earn Money at Home », «Big Money and Fast Sales »... Il s'agit bien d'offrir à un public peu qualifié la promesse d'une réussite rapide via des formations de faible niveau. Or, même s'il s'agit de promettre des emplois de comptable ou d'ouvrier qualifié, les publicités ne sont pas étrangères à l'esprit d'aventure qui anime le périodique. Nous en voudrions pour preuve la place considérable prise par les annonces de formation mettant en avant la promesse de voyages : "See the World and Earn Big Money!", " Big Opportunities Are Open Now On Land and On Sea», "Travel for Uncle Sam». Certaines annonces sont plus claires encore dans leurs promesses d'aventures: "Do you enjoy outdoor life close to Nature? Get Forest Ranger Job »; c'est le cas également de cette offre quelque peu loufoque pour devenir expert en empreintes digitales : «To command the highest fees, the Secret Service man must also be a Finger Print Expert.» Dans de telles 
formules se confondent les imaginaires romanesques et les désirs de réussite sociale. Les publicités retrouvent, mais dans une version plus prosaïque, plus réaliste (lors même que la plupart de ces annonces sont des attrape-nigauds), les rêves d'accomplissement professionnel qui transparaissent dans les pages d'« Ask Adventure », marquées par l'imaginaire du pionnier ${ }^{51}$.

C'est bien un mélange entre l'esprit d'aventure et la conquête d'un destin personnel qui est mis en avant dans les publicités. On s'en rend mieux compte encore quand on observe la série d'annonces de l'International Correspondence School confrontant, avec des variantes, un personnage ayant gravi les échelons après avoir décidé de se former et un de ses collègues, condamné à rester au bas de l'échelle sociale : «Don't tell me you never had a chance » dit, sur une des publicités, l'ambitieux récompensé à l'ancien collègue qui n'a su s'élever et semble le jalouser ${ }^{52}$. On reconnait là le motif du « rags to riches » qu'avait rendu fameux à la fin du xixe siècle Horatio Alger, en narrant les aventures de jeunes garçons pauvres s'élevant dans la société à force de persévérance et d'audace ${ }^{53}$.

L'imaginaire du self made man unit les publicités, les récits d'aventures et les rubriques du magazine. Les chercheurs d'or, les héros aux nerfs d'acier transportant des marchandises dans leur cargo, les pionniers du far west, les chasseurs de trésor sont récompensés suivant une logique « rags to riches», qui voit récompensés les efforts des audacieux et favorise une forme d'individualisme caractéristique du roman d'aventures à l'américaine. Et c'est cette même logique que recherchent les lecteurs qui souhaitent partir à l'aventure, mettant en œuvre un projet de vie fondé sur la confiance dans la volonté et le courage : «I would like some information about homesteading in Canada. How much land can one man take up? When is the best time in the year to come up there?54 »; « there are two of us who will make this trip [to the west coast of South America]. Our training and experience consist of many years of roughing it in the Yukon territory and Alaska [...]. The primary object of this proposed trip would be to prospect for gold and silver 55 . " Parfois la lettre développe explicitement un programme «rags to riches », à l'instar de celle qu'envoie un électricien envisageant d'aller s'installer en Inde. "I am 36 years old, strong, and not afraid of work », commence ce dernier, définissant les qualités requises pour réussir dans le monde. «Am total abstainer from liquor and tobacco» continue-t-il, dessinant ainsi un portrait moral du self made man. Car c'est bien une telle ambition qui détermine le projet du correspondant : «to work his way up from a minor job ${ }^{56}$ ». Si l'aventure fait rêver, 
c'est qu'elle reformule le rêve libéral du héros «rags to riches » ou du pionnier de gagner sa place dans le monde grâce à ses seuls mérites. Et de fait, par-delà la variété des programmes (partir en Inde, exploiter une vaste portion de terres sauvages, chercher de l'or), on reconnait toujours dans ces projets ambitieux l'idée que la détermination peut venir à bout des plus grandes difficultés - idée présente à la fois dans les publicités de l'International Correspondence School et dans les romans d'aventures à l'américaine.

Reste que, comme nous l'avons vu, ces entreprises périlleuses qu'envisagent les aventuriers en herbe sur le modèle des héros de papier sont généralement découragées par les spécialistes, qui démontrent sans peine que le monde de l'aventure n'existe plus ou que les espaces sauvages sont trop dangereux pour les expéditions improvisées ${ }^{57}$. Même le vieux fantasme du troupeau, qui avait nourri l'imaginaire des cowboys, suppose désormais un capital trop important s'il veut avoir une chance d'aboutir ${ }^{58}$. Mais les héros audacieux de l'International Correspondence School peuvent, eux, espérer devenir contremaitres ou managers à force d'études et de travail. Opposant aux récits un modèle de réussite certes moins romantique, mais plus en phase avec la réalité sociale contemporaine, les publicités semblent ainsi indiquer que le destin des Américains ne s'associe plus tant au charriot des pionniers qu'aux efforts déployés par les cols bleus et blancs pour réussir dans les grandes métropoles.

C'est cette négociation entre un mythe structurant du xix ${ }^{\mathrm{e}}$ siècle et la réalité d'une Amérique dont l'avenir se situe désormais dans les usines et les villes que reflète l'écosystème du pulp Adventure. Certes, pour les lecteurs, l'aventure romanesque fournit encore une puissante grammaire de l'imagination, héritée de la culture des settlers, comme en témoigne la propension des fictions à contaminer la manière dont les lecteurs se rêvent un avenir aventureux. Mais, pour beaucoup, cet imaginaire n'existe sans doute que le temps de la lecture. Bien sûr, dans les pages des magazines, on projette toujours de partir; bien sûr, certains correspondants se présentent comme de véritables bourlingueurs; bien sûr encore, les auteurs eux-mêmes insistent sur la manière dont leurs expériences aventureuses ont pu nourrir leurs œuvres. Mais, pour tous ceux qui n'écrivent pas au magazine, autrement dit pour la majorité silencieuse, cette expérience se résume sans doute à la communauté virtuelle d'un « Camp-Fire » à l'existence purement médiatique. Pour ceux-là, qui se contentent d'être simples lecteurs, cette communauté aussi est une fiction, et 
les projets audacieux qui l'animent restent imaginaires. Encore convient-il de noter qu'il s'agit d'une imagination qui n'est pas hétérogène au monde, une sorte d'horizon des possibles. Quand bien même l'aventure ne prendrait plus que la forme de rêveries à distance en vue d'hypothétiques voyages, elle n'en nourrit pas moins les représentations, et colonise indirectement la manière dont l'ouvrier, l'employé ou le jeune lecteur conçoivent leur existence. La nécessité de gravir les échelons devient alors une aventure épique reformulant, du côté de la réussite sociale, le vieux mythe pionnier. Au fil du temps d'ailleurs, les rubriques du «Camp-Fire» et d'« Ask Adventure» délaissent les projets de prospection ou d'expéditions sauvages pour laisser une place de plus en plus importante au tourisme extrême - voyage en canoë, trekking dans le Grand Nord ou expédition mexicaine. Et si, dans les années 1920, les lettres imaginant des entreprises hasardeuses sont nombreuses, dans les années 1930, l'aventure semble ne plus engager autant qu'avant un projet de vie. Ce sont alors les loisirs qui prolongent, dans l'existence, l'imaginaire des romans d'aventures. Ainsi le magazine suit-il au fil des années les transformations des imaginaires sociaux des lecteurs populaires américains : encore marqués dans leur correspondance par le mythe du pionnier qui a structuré les discours au xix $^{\mathrm{e}}$ siècle, ces lecteurs expérimentent sa reconfiguration suivant un modèle de réussite sociale lié au capitalisme industriel moderne et à la culture urbaine. Les rêveries aventureuses dans les pays lointains apparaissent alors comme la manifestation fantasmatique de cet imaginaire individualiste de la conquête sociale, qui continuent d'en représenter une forme noble, mais dont la relation à la réalité semble de plus en plus problématique.

\section{Conclusion}

L'exemple du pulp Adventure montre ainsi que si les logiques communicationnelles des fictions sérielles favorisent une évaluation des œuvres reposant sur des principes endogènes (ceux des architextes et de leurs conventions), celles-ci engagent néanmoins des questionnements qui touchent à la manière d'envisager le monde. La chose est en tout cas manifeste dans le temps de la lecture du magazine, et dans sa façon de produire une signification globale bien au-delà des seuls périmètres de la fiction. On voit bien, à travers les interactions entre les discours fictionnels et non fictionnels, combien les récits de genre mettent en jeu des fantasmes personnels, rêveries de loisirs extraordinaires et de conquête sociale. Cela signifie aussi que, malgré 
leur caractère stéréotypé, les œuvres sont perçues comme enracinées dans le monde - et c'est pour cette raison qu'elles peuvent trouver des prolongements dans des projets plus ou moins extravagants. Dans le cas d'Adventure, cet engagement dans l'existence est favorisé par des dispositifs propres au périodique et par des postures auctoriales jouant sur la confusion entre la biographie et les récits. Certes, les logiques du pulp, entre magazine et collection de genre, facilitent sans doute plus que d'autres supports de tels échanges, mais Adventure ne fait qu'accentuer un effet plus généralement produit par les œuvres à forte cohérence sérielle.

La deuxième remarque que l'on peut faire, c'est que la manière dont les productions de genre interagissent avec le monde est elle-même génériquement marquée. Dans la partie rédactionnelle du magazine, les centres d'intérêt partagés, les sujets abordés, les modalités d'écriture des lecteurs et des rédacteurs, la posture adoptée (le rôle médiatique et ses intertextes romanesques) et la façon de penser son existence s'inscrivent dans une forme de mystique de l'aventure, dont la cohérence "romanesque » apparait ici saillante dans la relation introduite entre les fictions et les textes non fictionnels. C'est bien une conception globale du monde et de l'existence qui se fait jour dans Adventure, valorisant un esprit d'entreprise et un individualisme qu'exprime le vocabulaire du roman d'aventures. Or, si notre étude portait sur le roman d'aventures, on a vu que ces procédés se retrouvaient dans la partie rédactionnelle des autres genres de pulps (sentimentaux, science-fiction, western, etc.) qui privilégiait une représentation du monde, des centres d'intérêts et surtout des scénarios imaginaires commandés par le genre, mais affectant plus largement une façon d'envisager l'existence. Si l'on tient compte du fait que les genres médiatiques n'existent que comme résultats des échanges polyphoniques entre une multitude d'œuvres situées en un temps et un lieu et de leur négociation avec l'héritage des conventions sérielles, il est alors permis de penser que le développement d'un genre médiatique opéré par une communauté (ou sa transformation à un moment donné) est l'expression de préoccupations collectives qui dépassent largement le champ de la fiction. Manière de considérer le monde, de lui donner sens, structure de signification et axiologie, formulation des tensions du temps, le genre apparaît ainsi comme un mode d'expression des représentations d'une société et des tensions qui les traversent, puisqu'il est né naturellement de la multitude des discours qui le constituent. À l'inverse, chaque œuvre de genre, chaque instanciation des logiques génériques (par 
exemple un périodique ou une collection à fort ancrage générique) peut alors être interprétée comme la formulation située de ces tensions, une manière de se positionner par rapport au discours collectif du genre. Dès lors, se pencher sur les différents genres d'une époque en tenant compte de leurs variations et des contextes interdiscursifs qui les portent reviendrait à interroger la façon dont, à travers ces genres et leurs modèles de narration, une culture donnée organise en systèmes de signification (mais en systèmes instables et traversés de contradictions dialogiques) certaines des préoccupations majeures qui sont les siennes.

C'est ce que nous a semblé révéler Adventure. Ce magazine montre en effet combien le discours sur l'aventure qui se développe à la fois dans la partie rédactionnelle et dans les récits contribue à nourrir un imaginaire de la réussite individuelle et de la conquête sociale dont le genre offrirait une version fantasmatique. Dans sa relation à l'aventure, le lecteur oscille entre une reconfiguration romanesque de l'imaginaire du self made man et une adhésion, au premier degré, aux valeurs du pionnier. Ce goût pour le récit d'aventures dans les confins et pour les projets d'expéditions extraordinaires compense le sentiment d'un déclin du mythe de l'Ouest, qui n'existe plus, dans le périodique, qu'à l'état de mémoire et qui a laissé place, dans les publicités, à des préoccupations plus réalistes de conquête sociale en phase avec les logiques du capitalisme moderne. Loin des imaginaires patriotiques ou coloniaux des Européens, l'aventure sublime ainsi la question de la réussite individuelle, via une distance exotique qui semble toujours plus grande par rapport à ce monde de cols blancs et bleus qui est désormais celui des Américains.

Matthieu Letourneux est professeur à l'Université Paris-Nanterre et membre du CSLF. Spécialiste des cultures sérielles et médiatiques des xix et $\mathrm{xx}^{\mathrm{e}}$ siècles et de la littérature pour la jeunesse, il vient de faire paraitre un essai sur la sérialité, Fictions à la chaîne, Seuil, "Poétique », 2017. Il a publié auparavant Cinéma, premiers crimes (avec A. Carou, Paris Bibliothèques, 2015), Fantômas! Biographie d'un criminel imaginaire (avec L. Artiaga, Les Prairies ordinaires, 2013), La Librairie Tallandier: Histoire d'une grande maison d'édition populaire (1870-2000) (avec J.-Y. Mollier, Nouveau Monde, 2011) et Le Roman d'aventures, 1870-1930 (PULIM, 2010). Il a dirigé ou codirigé une dizaine de publications collectives sur les littératures de jeunesse ou les cultures médiatiques. Il a réédité et préfacé Le Coureur des bois de Gabriel Ferry (Phébus), On vole des enfants à Paris de Louis Forest (Le Masque), et chez Bouquins des ouvres de Gustave 
Aimard, Emilio Salgari, Eugène Sue et la série Fantômas (avec L. Artiaga). Il est rédacteur en chef de la revue Belphégor (http://belphegor.revues.org/).

\section{Notes}

1 On trouve une description de la place des pulps dans l'écosystème éditorial dans R. D. Mullen, «From Standard Magazines to Pulps and Big Slicks: A Note on the History of US General and Fiction Magazines », Science Fiction Studies, vol. 22, n 1, mars 1995.

2 Selon R. D. Mullen, il fait partie des dix premiers pulps créés; R. D. Mullen, «From Standard Magazines to Pulps and Big Slicks: A Note on the History of US General and Fiction Magazines », Science Fiction Studies, vol. 22, n 1, mars 1995.

3 «No. 1 Pulp», Time Magazine, 21 October 1935, cité par Richard Bleiler, "A History of Adventure Magazine », The Index to Adventure Magazine, San Bernardino, Starmont House, The Borgo Press, 1990.

${ }^{4}$ Il le fait sur un mode volontiers ironique, surjouant les stéréotypes présents dans le récit: "All Swedes spend their spare moments drinking, in fact my dear mother can not go to the Swedish Baptist Church on Sunday without taking at least a quart of moonshine "; sans titre, Adventure, vol. 42, $\mathrm{n}^{\circ} 2$, 20 août 1923.

${ }^{5}$ La critique est adressée à Alan Le May, dans son récit « Old Father of Waters »; « Port and

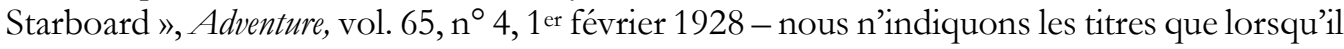
y en a.

${ }^{6}$ "I have long been preparing a protest to Adventure's method of deleting the rough words of rough men in rough situations »; " Rough Words and Rough Men », Adventure, vol. 64, n 5, 15 novembre 1927.

7 Adventure, vol. 49, $\mathrm{n}^{\circ}$ 4, 10 novembre 1924. Le livre de Hough avait été violemment critiqué par Stuart Henry. Une partie de ces critiques a été reproduite dans l'appendice de Conquering Our Great American Plains (1930).

8 On trouve un certain nombre d'informations sur ces spécialistes dans Richard Bleiler, «A History of Adventure Magazine », The Index to Adventure Magazine, San Bernardino, Starmont House, The Borgo Press, 1990.

9 Le sujet revient à plusieurs reprises. On citera par exemple : "Our Uncharted River », Adventure, vol. 42, n 2, 20 août 1923; «A Canoe on the Mississippi», Adventure, vol. 42, n 6, 30 septembre 1923 , etc.

${ }^{10}$ Sans titre, Adventure, vol. 89, n $5,1^{\text {e }}$ octobre 1934.

11 «Jungle Travel in New Guinea », Adventure, vol. 42, n 3, 30 août 1923.

12 Par exemple dans l'échange titré «Prospecting the Andes », Adventure, vol. 23, n 5, 3 décembre 1919; ou dans «Making a Mine », Adventure, vol. 42, n² 2, 20 août 1923. 
${ }^{13}$ Malgré les changements de direction à la tête du périodique et malgré la Seconde Guerre mondiale, la structure rédactionnelle d'Adventure reste étonnamment stable, comme d'ailleurs son choix de conserver pour ses récits une conception large de l'aventure (mêlant récits western, exotiques et historiques), quand les pulps ont eu tendance à rechercher une spécialisation de plus en plus grande dans l'espoir d'être identifiables au sein d'une offre pléthorique. Il faut attendre les années 1950, et la crise qui traverse l'ensemble des pulps, pour que le périodique change de formule et adopte un format proche des « male magazines». Encore faut-il noter que les rubriques «Camp-Fire » et « Ask Adventure » sont toujours présentes dans le sommaire du périodique dans les années 1960, alors que celui-ci a pleinement adopté la formule des «male magazines» (articles mêlant sexe et violence et photos dénudées). Mais leur contenu s’est sensiblement transformé.

14 «Adventure Travel Association », Adventure, vol. 60, n 4, 23 novembre 1926.

15 Ceux-ci, proposés régulièrement dans le périodique, sont soumis aux lecteurs dans le «Camp-Fire » du volume 22, n 3, 3 août 1919.

${ }^{16}$ Adventure n'est pas le seul à chercher à organiser une communauté de lecteurs en exploitant les sujets des fictions dans la partie rédactionnelle. Dans les années 1920 et 1930, certains pulps sentimentaux (comme Love Story Magazine) offraient ainsi courrier du cœur, petites annonces de rencontre, conseils sentimentaux et astrologie, certains pulps criminels (comme Detective Fiction Weekly), des énigmes et codes à déchiffrer mettant en jeu le courrier des lecteurs, les pulps western (comme Ace-High Magazine), des bourses aux échanges pour les collectionneurs d'americana et des conseils sur les armes à feu, et les pulps de science-fiction (comme Astounding Stories), des échanges avec les lecteurs autour de la possibilité scientifique de certaines inventions présentes dans les récits.

17 Ainsi l'éditeur insiste-t-il sur le fait que les services qu'il offre sont "pour l'essentiel gratuits » et qu' « ils engagent beaucoup de temps, de travail et de dépenses », mais que « dans la mesure où il peut aider, il est prêt et désireux de le faire » (" they involve much time, work and expense on our part [...] Whenever we can help we're ready and willing to try »); "Various Practical Services to Any Reader», Adventure, vol. 42, n 3, 30 août 1923. Nous traduisons.

18 «We are drawn together by a common liking for the strong, clean things of out-of-doors, for word from the earth's far places, for man in action instead of caged by circumstance. The spirit of adventure lives in all men; the rest is chance »; "The Camp-Fire», Adventure, vol. 33, n 3, 28 février 1922. Nous traduisons. Le texte apparait généralement en tête de rubrique.

${ }^{19}$ Marie-Ève Thérenty, La littérature an quotidien, Paris, Seuil, « Poétique », 2007.

${ }^{20}$ Sylvain Venayre, La gloire de l'aventure, Paris, Aubier, 2002.

${ }^{21}$ Voir note 12.

${ }^{22}$ Preuve de l'influence de Jack London sur le journal, un lecteur propose peu après sa mort de lancer une souscription dans le journal pour lui dresser un monument; Adventure, vol. 18, n 5,3 septembre 1918.

23 Par exemple dans Alan Le May, "Baldy at the Brink», Adventure, vol. 60, n 4, 23 novembre 1926; ou dans R. H. Lowdermilk, «Three Wise Men », Adventure, vol. 23, n 5, 3 décembre 1919. C'est dans ce dernier numéro qu'un lecteur demande à quelles conditions il peut prospecter dans les Andes.

Vol. 10, $\mathrm{n}^{\circ} 1 \mid$ Automne 2018

«Voyager dans la modernité : des moyens techniques aux support imprimés de l'aventure » 
${ }^{24}$ Par exemple dans tel projet de partir quelques années au Nigéria en vivant de chasse, Adventure, vol. 42, $\mathrm{n}^{\circ}$ 2, 20 août 1923; ou dans cette demande pour devenir mécanicien dans les îles des mers du Sud, Adventure, vol. 74, n 1, 15 mars 1930.

${ }^{25}$ Cette influence du roman européen dans Adventure peut se lire dans la présence des auteurs anglais (T. Mundy, R. Sabatini, H. Lamb, etc.) au sein du périodique. La logique d'américanisation peut quant à elle se lire dans la méfiance à l'égard des imaginaires coloniaux (c'est le sens des inquiétudes de certains lecteurs quant au prosélytisme britannique de Talbot Mundy, auxquelles répond le périodique dans le volume $42, n^{\circ} 2$, du 20 août 1923).

${ }^{26}$ « On Short Allowance », Adventure, vol. 18, n² 2, 18 juillet 1918.

${ }^{27}$ « The Pearls of Torres », Adventure, vol. 18, n 2, 18 juillet 1918.

28 «The Mutiny », Adventure, vol. 33, n 3, 28 février 1922.

${ }^{29}$ Richard Slotkin, Regeneration Through Violence et Gunfighter Nation, Norman, University of Oklahoma Press, 1985 et 1998.

${ }^{30}$ Dans le volume 42, numéro 2 (20 août 1923), W. C. Tuttle insiste par exemple sur le fait que " "Powder Law" est basé sur des faits, et nombre des incidents qu'il conte ont été empruntés à la vie elle-même », affirmant en outre, sans doute dans un souci plus général de légitimation, que son père avait été sheriff à Dawson. Nous traduisons.

${ }^{31}$ Sans titre, Adventure, vol. 42, n 3, 30 août 1923.

${ }^{32}$ Sans titre, Adventure, vol. 60, n 3, 8 novembre 1926.

33 Respectivement dans Adventure, vol. 49, $\mathrm{n}^{\circ}$ 4, 10 novembre 1924, et vol. 68, $\mathrm{n}^{\circ}$ 5, 15 novembre 1928.

${ }^{34}$ Sur le Mississippi, on l'a vu, mais aussi en Ontario, etc.

35 « Telegraph Creek », Adventure, vol. 42, n 2, 20 août 1923.

36 "I want to go to as many of the out of way spots and still remain on a motorcycle. I want to stop beside the road for the night and prepare a supper without seeing an advertising sign.» La réponse est pour le moins décevante dans son réalisme: "Your gasoline supply, alone, will present a major problem. Also there will be stretches of mountain trail, jungle, etc., where you will have to bire ox-cart transportation for your motorcycle. You may even have to dismantle it and pack it by burro »; " Motorcycling in Old Mexico ", Adventure, vol. 91, n² 2, 15 février 1935.

${ }^{37}$ «Cruising Georgian Bay », Adventure, vol. 42, n 3, 30 août 1923.

38 «Too Much Territory», Adventure, vol. 49, n 4, 10 novembre 1924.

39 « Nigeria », Adventure, vol. 42, n 2, 20 août 1923.

${ }^{40}$ Nous citerons encore deux exemples de réponses réalistes opposées aux projets des lecteurs : un expert d'« Ask Adventure » explique qu'il n'est guère raisonnable d'improviser 
un système de livraison de marchandises dans les Mers du Sud (« Your idea of sailing a 500-ton registered schooner into the South Seas is a good one, but the chances are ten to one that the venture (or is it an adventure?) will go down in the log as a financial catastrophe »); " Free-lance Trading in the South Seas ", Adventure, vol. 33, n 3, 28 février 1922. Un autre souligne qu'il n'est pas possible pour un Blanc de trouver un emploi en Afrique de l'Est s'il n'a pas été engagé avant son départ; Adventure, vol. 22, n 3, 3 août 1919.

${ }^{41}$ Ce que nous appelons un ton hardboiled est un langage familier employé avec affectation et destiné à manifester une posture virile, comme dans la phrase suivante "Knew a fellow named John Parr, extra gang-foreman and all round track»; "Automobiling in Colombia ", Adventure, vol. 53, n 5, 20 juillet 1925.

${ }^{42}$ « Motoring across the Continent », Adventure, vol. 53, n 3, 30 juin 1925.

${ }^{43}$ C'est à travers leurs exploits de guerre que se présentent par exemple les écrivains Théodore Fredenburgh (juillet 1934), Kenneth B. Collings (15 février 1935), ou Henry Church (1er mai 1935).

${ }^{44}$ Voir par exemple la présentation de soi que fait William S. Wells : « How come I to start writing stories? Well, I got the idea mostly from my father, William Wells, who left a vacant seat at this same Camp-Fire when he was called to the "last round-up" last March. I got from bim enough of the real dope on the old West, which together with some of my own experiences here and there, sort of gave the idea a boost. Then, too, it's come to the point where a fellow has to make a living one way or another $» ;$ Adventure, vol. $91, \mathrm{n}^{\circ} 2,15$ février 1935. On est frappé par le mimétisme entre le parler et la posture de l'auteur et ceux des personnages d'Adventure.

${ }^{45}$ Pour José Luis Diaz, la scénographie auctoriale désigne les rôles et les postures attendus, à une époque et dans un cadre donnés, pour l'écrivain; J. L. Diaz, Écrivain imaginaire: scénographies auctoriales à l'époque romantique, Paris, Champion, 2007.

${ }^{46}$ Sans titre, Adventure, vol. 60, n 3, 8 novembre 1926.

${ }^{47}$ Sans titre, Adventure, vol. 60, n 3, 8 novembre 1926.

48 Ainsi de Foster Harris qui présente en ces termes son récit: «But, since "Can Roller" deals with such matters, I will claim to know an Indian or two and a little bit about boom oil towns, their John Laws and their shooters, can, oil or otherwise. I was born in the Chickasaw Nation, back when. Lived in New Mexico several years. And I've been at least exposed to oil game atmosphere nearly all my life » (1er mai 1936). Et le Captain William Alford introduit son récit «Life on a Hell Ship» en insistant sur sa connaissance de la mer: "At 14 I went to sea as apprentice on the Charlotte Young, a threemasted barque [...] I have followed the sea 33 years, 17 of which were in sail » (15 février 1935).

49 "As I look back now and attempt an analysis I am moved to remark that it has been a large part of my education. Since I have so far been unable to travel as extensively as I would like, I realize that I have become somewhat familiar with the lives and customs of people in strange places. I am a close reader of Camp-Fire and the other departments of Adventure. Because these discussions and arguments are carried on between men who base their statements upon personal experiences, I have come to accept stories and articles alike as authoritative »; Adventure, vol. 64, n 5, 15 novembre 1927. Nous traduisons.

${ }^{50}$ Matthieu Letourneux, Fictions à la chaîne. Littératures sérielles et culture médiatique, Paris, Seuil, «Poétique », 2017.

Vol. 10, $\mathrm{n}^{\circ} 1 \mid$ Automne 2018

«Voyager dans la modernité : des moyens techniques aux support imprimés de l'aventure » 
${ }^{51} \mathrm{Il}$ est évident que la plupart de ces publicités n'étaient pas réservées à Adventure, mais leur accumulation et les échos qu'elles tissent avec les autres pages du journal font sens. En outre, une comparaison rapide avec des pulps spécialisés dans d'autres genres démontre des effets de spécialisation thématique des publicités qui contribuaient à l'imaginaire de ces périodiques : importance des publicités technologiques dans Astounding, vente de revolver et publicités pour devenir policier ou détective privé dans Detective Story Magazine, etc.

52 Adventure, vol. 22, n 3, 3 août 1919.

${ }^{53}$ Richard Bowerman, "Horatio Alger, Jr.; or, Adrift in the Myth of Rags to Riches », The Journal of American Culture, vol. 2, n 1, 1979. La circulation des imaginaires proposés par H. Alger dans les productions de romans d'aventures a sans doute été facilitée par le fait qu'il s'inspirait lui-même des romanciers d'aventures; Gary Scharnhorst, "Had Their Mothers Only Known: Horatio Alger, Jr., Rewrites Cooper, Melville, and Twain », The Journal of Popular Culture, vol. XV, n 3, 1981.

54 «Homesteading in Canada », Adventure, vol. 33, n 3, 28 février 1922.

55 « Prospecting the Andes », Adventure, vol. 23, n 5, 3 décembre 1919.

56 «Employment in India », Adventure, vol. 18, n² 2, 18 juillet 1918.

${ }^{57}$ C'est à tel point que, quand ils en viennent à encourager un projet de lecteur, les experts semblent en être les premiers surpris : "Your letter is one of the very few which I have received, in reply to which I can give some encouragement about employment in India »; « Employment in India », Adventure, vol. 18, $\mathrm{n}^{\circ}$ 2, 18 juillet 1918.

58 «Plantation », Adventure, vol. 68, n 5, 15 novembre 1928.

\section{Bibliographie}

Richard Bleiler, "A History of Adventure Magazine », The Index to Adventure Magazine, San Bernardino, Starmont House, The Borgo Press, 1990.

Richard Bowerman, «Horatio Alger, Jr.; or, Adrift in the Myth of Rags to Riches », The Journal of American Culture, vol. 2, n 1, 1979.

José Luis Diaz, Écrivain imaginaire: scénographies auctoriales à l'époque romantique, Paris, Champion, 2007.

Stuart Oliver Henry, Conquering our great American plains: a historical development, New York, E.P. Dutton, 1930.

Matthieu Letourneux, Fictions à la chaîne. Littératures sérielles et culture médiatique, Paris, Seuil, « Poétique », 2017.

R. D. Mullen, «From Standard Magazines to Pulps and Big Slicks: A Note on the History of US General and Fiction Magazines », Science Fiction Studies, vol. 22, n ${ }^{\circ} 1$, mars 1995. 
Gary Scharnhorst, « Had Their Mothers Only Known: Horatio Alger, Jr., Rewrites Cooper, Melville, and Twain », The Journal of Popular Culture, vol. XV, n 3, 1981.

Richard Slotkin, Regeneration Through Violence et Gunfighter Nation, Norman, University of Oklahoma Press, 1985 et 1998.

Marie-Ève Thérenty, La Littérature au quotidien, Paris, Seuil, « Poétique », 2007.

Sylvain Venayre, La Gloire de l'aventure, Paris, Aubier, 2002. 